M. Barros and B.-Y. Chen

Nagoya Math. J.

Vol. 108 (1987), 77-91

\title{
SPHERICAL SUBMANIFOLDS WHICH ARE OF 2-TYPE VIA THE SECOND STANDARD IMMERSION OF THE SPHERE
}

\author{
MANUEL BARROS AND BANG-YEN CHEN
}

\section{§1. Introduction}

Let $S^{m}(r)$ be an $m$-sphere of constant sectional curvature $1 / r^{2}$ and $M$ an $n$-dimensional compact minimal submanifold of $S^{m}(r)$. If $S^{m}(r)$ is imbedded in $E^{m+1}$ by its first standard imbedding, then, by a well-known result of Takahashi [11], the Euclidean coordinate functions restricted to $M$ are eigenfunctions of $\Delta$ on $M$ with the same eigenvalue $n / r^{2}$. Moreover, the center of mass of $M$ in $E^{m+1}$ coincides with the center of the hypersphere $S^{m}(r)$ in $E^{m+1}$. Thus, $M$ is mass-symmetric in $S^{m}(r) \subset E^{m+1}$. Consequently, we see that if one wants to study the spectral geometry of a submanifold of $S^{m}(r)$, it is natural to immerse $S^{m}(r)$ by its $k$-th standard immersion, in particular, by its second standard immersion.

In [9], A. Ros has used this idea to study compact minimal submanifolds of $S^{m}(r)$ via the second standard immersion. In [9], he obtained a formal characterization of a compact minimal submanifold $M$, fully in $S^{m}$, such that the Euclidean coordinate functions restricted to $M$ via the second standard immersion $f$ of $S^{m}$ are described by means of two different eigenvalues of $\Delta$, i.e., $M$ is of 2-type via $f$. He showed that such submanifolds are Einstein and mass-symmetric via $f$. However, he did not obtain any classification result for such submanifolds.

In this paper, we study compact submanifolds of a sphere which are mass-symmetric and of 2-type via the second standard immersion of the sphere. In Section 3, we obtain a generalization of Ros' characterization (Lemma 1). Some primary classifications are obtained in this section (Theorems 1 and 2). In Section 4, hypersurfaces of a sphere which are mass-symmetric and of 2-type via $f$ are completely classified (Theorem 3).

Received May 22, 1986. 
In Section 5, submanifolds of $S^{m}$ with "maximal possible" codimension are studied. In the last section, results in previous sections are applied to obtain a classification theorem of compact surfaces of $S^{m}$ which have the desired properties via $f$.

\section{§. Basics}

Let $x: M \rightarrow E^{m}$ be an isometric immersion of a compact, connected, $n$-dimensional, Riemannian manifold $M$ into a Euclidean $m$-space. Denote by $\operatorname{Spec}(M)=\left\{0=\lambda_{0}<\lambda_{1}<\ldots<\lambda_{k}<\cdots \uparrow \infty\right\}$ the spectrum of $\Delta$ acting on differentiable functions in $C^{\infty}(M)$. If we extend the Laplace-Beltrami operator $\Delta$ to $E^{m}$-valued functions on $M$ in a natural fashion, then, we have the following spectral decomposition of $x$ (in $L^{2}$-sense) (cf. $[1,3,5$, $6,9])$ :

$$
x=x_{0}+\sum_{t=1}^{\infty} x_{t}, \Delta x_{t}=\lambda_{t} x_{t}, x_{t}: M \longrightarrow E^{m},
$$

where $x_{0}$ is the center of mass of $M$ in $E^{m}$. The submanifold $M$ is said to be of finite type if the spectral decomposition of $x$ consists of only finitely many nonzero terms. More precisely, $M$ is said to be of $k$-type if there are exactly $k$ nonzero $x_{t}$ 's $(t \geqq 1)$ in the decomposition of $x([5,6])$.

From the Takahashi Theorem [11] we know that $M$ is of 1-type if and only if $M$ is a minimal submanifold of a hypersphere $S^{m-1}(r)$ of $E^{m}$. In this case, $M$ is mass-symmetric in $S^{m-1}(r) \subset E^{m}$, i.e., the center of mass of $M$ in $E^{m}$ coincides with the center of $S^{m-1}(r)$ in $E^{m}$ (cf. [6]).

Let $x: M \rightarrow E^{m}$ be a 2-type submanifold with mean curvature vector $H$. Then we have

$$
x=x_{0}+x_{p}+x_{q}, \quad \Delta x_{p}=\lambda_{p} x_{p}, \quad \Delta x_{q}=\lambda_{q} x_{q}
$$

for some integers $p, q(q>p \geqq 1)$. Since $\Delta x=-n H$, (2.2) implies

$$
\Delta H=b H+e\left(x-x_{0}\right),
$$

where $b=\lambda_{p}+\lambda_{q}$ and $e=\lambda_{p} \lambda_{q} / n$.

On $E^{m}$ we consider an inner product $\left\langle\right.$, given by $\langle u, v\rangle=u \cdot v^{t}$ for any $u, v \in E^{m}$, where each vector in $E^{m}$ is regarded as a row matrix and $v^{t}$ is the transpose of $v$. Let $r>0$. Then the sphere $S^{m-1}(r)=\left\{x \in E^{m} \mid\right.$ $\left.\langle x, x\rangle=r^{2}\right\}$ with the induced metric has constant sectional curvature $1 / r^{2}$. Let $S M(m)=\left\{P \in \operatorname{gl}(m ; R) \mid P^{t}=P\right\}$ be the space of symmetric $m$ by $m$ matrices over $R$ endowed with the metric $g(P, Q)=\left(1 / 2 r^{2}\right) \operatorname{tr}(P Q)$ for 
$P, Q \in S M(m)$. Consider the mapping $f: S^{m}(r) \rightarrow S M(m+1)$ defined by $f(u)=u^{t} \cdot u$. Then $f$ is an isometric immersion which is in fact the second standard immersion of $S^{m}(r)$. The image $f\left(S^{m}(r)\right)$ is a real projective space which lies fully in an $(m+m(m+1) / 2)$-dimensional linear space of $S M(m+1)$. We call $f\left(S^{m}(r)\right)$ a Veronese submanifold.

For each point $u \in S^{m}(r)$, the normal space of $S^{m}(r)$ in $S M(m+1)$ at $u$ (or more precisely at $f(u)$ ) is given by

$$
T_{u}^{\perp}\left(S^{m}(r)\right)=\{P \in S M(m+1) \mid u P=\mu u \text { for some } \mu \in \boldsymbol{R}\} .
$$

In particular, we have $f(u) \in T_{u}^{\perp}\left(S^{m}(r)\right)$.

If $\bar{\sigma}$ is the second fundamental form of $f$, we have

$$
\bar{\sigma}(X, Y)=X^{t} \cdot Y+Y^{t} \cdot X-\left(2 / r^{2}\right)\langle X, Y\rangle f(u)
$$

for $X, Y$ in $T_{u}\left(S^{m}(r)\right)$. It is known that $\bar{\sigma}$ is parallel and it satisfies

$$
\begin{gathered}
g(\bar{\sigma}(X, Y), \bar{\sigma}(V, W))=\left(1 / r^{2}\right)\{2\langle X, Y\rangle\langle V, W\rangle+\langle X, V\rangle\langle Y, W\rangle \\
+\langle X, W\rangle\langle Y, V\rangle\}, \\
g(\bar{\sigma}(X, Y), f(u))=-\langle X, Y\rangle, \quad g(\bar{\sigma}(X, Y), I)=0, \\
\bar{A}_{\bar{\sigma}(X, Y)} V=\left(1 / r^{2}\right)\{2\langle X, Y\rangle V+\langle X, V\rangle Y+\langle Y, V\rangle X\},
\end{gathered}
$$

where $\bar{A}$ is the Weingarten map of $f, X, Y, V, W \in T_{u}\left(S^{m}(r)\right)$, and $I$ the identity matrix.

It is known that $S^{m}(r)$ is immersed by the second standard immersion $f$ as a minimal submanifold of a hypersphere of $S M(m+1)$ centered at $r^{2} I /(m+1)$ and with radius $\left(r^{2} m / 2(m+1)\right)^{1 / 2}$. For more details, see $[6,9$, 10].

In the following, we simply denote $S^{m}(1)$ by $S^{m}$.

\section{§3. Submanifolds of $S^{m}$ which are of 2-type via $f$}

Let $\psi: M \rightarrow S^{m}$ be an isometric immersion of $M$ into $S^{m}$. We denote by $\sigma^{\prime}, H^{\prime}$ and $A$ the second fundamental form, the mean curvature vector and the Weingarten map of $\psi$, respectively. Denote by $\nabla$ and $\bar{\nabla}$ the LeviCivita connections on $M$ and $S^{m}$, respectively, and by $D$ the normal connection of $\psi$.

We consider the isometric immersion $x: M \rightarrow S M(m+1)$ defined by

$$
x=f \circ \psi: M \stackrel{\psi}{\longrightarrow} S^{m} \stackrel{f}{\longrightarrow} S M(m+1) .
$$

Then the mean curvature vector $H$ of $x$ satisfies 


$$
H=H^{\prime}+\frac{1}{n} \sum_{i=1}^{n} \bar{\sigma}\left(E_{i}, E_{i}\right)
$$

where $H^{\prime}$ is identified with the image $f_{*} H^{\prime}$ of $H^{\prime}$ under $f_{*}$ and $E_{1}, \cdots, E_{n}$ is an orthonormal frame tangent to $M$.

Let $u$ be an arbitrary point in $M$. We may assume that $\nabla_{E_{j}} E_{i}=0$ at $u$. We compute $\Delta H^{\prime}$ at $u$.

$$
\begin{aligned}
\left(\Delta H^{\prime}\right)(u)= & -\sum_{i=1}^{n} E_{i} E_{i} H^{\prime} \\
= & \sum_{i=1}^{n}\left\{\bar{\nabla}_{E_{i}} A_{H^{\prime}} E_{i}+\bar{\sigma}\left(E_{i}, A_{H^{\prime}} E_{i}\right)-\bar{\nabla}_{E_{i}} D_{E_{i}} H^{\prime}\right. \\
& \left.-\bar{\sigma}\left(E_{i}, D_{E_{i}} H^{\prime}\right)+\bar{A}_{\bar{\sigma}\left(E_{i}, H^{\prime}\right)} E_{i}-\bar{D}_{E_{i}} \bar{\sigma}\left(E_{i}, H^{\prime}\right)\right\},
\end{aligned}
$$

where $\bar{D}$ denotes the normal connection of $f$. By applying (2.8) and the fact that $\bar{\sigma}$ is parallel, we find

$$
\begin{aligned}
\left(\Delta H^{\prime}\right)(u)= & \Delta^{D} H^{\prime}+\operatorname{tr}\left(\bar{\nabla} A_{H^{\prime}}\right)+\sum \sigma^{\prime}\left(E_{i}, A_{H} E_{i}\right)+2 \sum \bar{\sigma}\left(E_{i}, A_{H H^{\prime}} E_{i}\right) \\
& -2 \sum \bar{\sigma}\left(E_{i}, D_{E_{i}} H^{\prime}\right)+n H^{\prime}-n \bar{\sigma}\left(H^{\prime}, H^{\prime}\right)
\end{aligned}
$$

where $\Delta^{D}$ is the Laplacian with respect to the normal connection $D$ and

$$
\operatorname{tr}\left(\bar{\nabla} A_{H^{\prime}}\right)=\sum\left(\nabla_{E_{\imath}} A_{H^{\prime}}\right) E_{i}+\sum A_{D_{E_{i} H^{\prime}}} E_{i} .
$$

For each point $u$ in $M$, we choose an orthonormal basis $\left\{\xi_{n+1}, \cdots, \xi_{m}\right\}$ of the normal space of $M$ is $S^{m}$ at $u$ such that $\xi_{n+1}$ is parallel to $H^{\prime}$ at $u$ (if $H^{\prime}=0$ at $u$, any orthonormal frame satisfies this condition). Simply denote $A_{\xi_{r}}(r=n+1, \cdots, m)$ by $A_{r}$. We have

$$
\sum_{i=1}^{n} \sigma^{\prime}\left(E_{i}, A_{H^{\prime}} E_{i}\right)=\left|A_{n+1}\right|^{2} H^{\prime}+\mathfrak{U}^{\prime}\left(H^{\prime}\right)
$$

where $\mathfrak{U}^{\prime}\left(H^{\prime}\right)=\sum_{r=n+2}^{m} \operatorname{tr}\left(A_{H^{\prime}} A_{r}\right) \xi_{r}$ is the so-called allied mean curvature vector of $M$ in $S^{m}$. It is clear that if $H^{\prime}=0$ at $u$, then $\mathfrak{I}^{\prime}\left(H^{\prime}\right)=\left|A_{n+1}\right|^{2} H^{\prime}$ $=0$ at $u$. It is easy to see that $\mathfrak{U}^{\prime}\left(H^{\prime}\right)$ and both sides of (3.4) are independent of the choice of $\xi_{n+1}, \cdots, \xi_{m}$ such that $\xi_{n+1}$ is parallel to $H^{\prime}$. By combining (3.2) and (3.4) we obtain

$$
\begin{aligned}
\left(\Delta H^{\prime}\right)(u)= & \Delta^{D} H^{\prime}+\operatorname{tr}\left(\bar{\nabla} A_{H^{\prime}}\right)+\left(\left|A_{n+1}\right|^{2}+n\right) H^{\prime}+\mathfrak{U}^{\prime}\left(H^{\prime}\right) \\
& +2 \sum \bar{\sigma}\left(E_{i}, A_{H^{\prime}} E_{i}\right)-2 \sum \bar{\sigma}\left(E_{i}, D_{E_{i}} H^{\prime}\right)-n \bar{\sigma}\left(H^{\prime}, H^{\prime}\right) .
\end{aligned}
$$

On the other hand, from (2.6), (2.7) and parallelism of $\bar{\sigma}$, we have 


$$
\begin{aligned}
& \frac{1}{n} \Delta\left(\sum_{i=1}^{n} \bar{\sigma}\left(E_{i}, E_{i}\right)\right)(u)=2(n+2) H^{\prime}+\frac{2}{n}(n+1) \sum_{j} \bar{\sigma}\left(E_{j}, E_{j}\right) \\
& \quad+\frac{2}{n} \sum_{i, j} \bar{\sigma}\left(A_{\sigma^{\prime}\left(E_{i}, E_{j}\right)} E_{j}, E_{i}\right)-\frac{2}{n} \sum_{i, j} \bar{\sigma}\left(\sigma^{\prime}\left(E_{i}, E_{j}\right), \sigma^{\prime}\left(E_{i}, E_{j}\right)\right) \\
& \quad-\frac{2}{n} \sum_{i, j} \bar{\sigma}\left(\left(\tilde{\nabla} \sigma^{\prime}\right)\left(E_{i}, E_{j}, E_{j}\right), E_{i}\right),
\end{aligned}
$$

where $\tilde{\nabla} \sigma^{\prime}$ denotes the covariant derivative of $\sigma^{\prime}$. From Codazzi's equation, we have

$$
\sum\left(\tilde{\nabla} \sigma^{\prime}\right)\left(E_{i}, E_{j}, E_{j}\right)=n D_{E_{i}} H^{\prime} .
$$

Thus, we obtain, from (3.1), (3.5), (3.6) and (3.7),

$$
\begin{aligned}
& (\Delta H)(u)=\Delta^{D} H^{\prime}+\operatorname{tr}\left(\bar{\nabla} A_{H^{\prime}}\right)+\mathfrak{U}^{\prime}\left(H^{\prime}\right)+\left(\left\|A_{n+1}\right\|^{2}+3 n+4\right) H^{\prime} \\
& +\frac{2(n+1)}{n} \sum_{j} \bar{\sigma}\left(E_{j}, E_{j}\right)+2 \sum_{i} \bar{\sigma}\left(E_{i}, A_{H^{\prime}} E_{i}\right) \\
& +\frac{2}{n} \sum_{i, j} \bar{\sigma}\left(A_{\sigma^{\prime}\left(E_{i}, E_{j}\right)} E_{i}, E_{j}\right)-4 \sum_{i} \bar{\sigma}\left(E_{i}, D_{E_{i}} H^{\prime}\right) \\
& -n \bar{\sigma}\left(H^{\prime}, H^{\prime}\right)-\frac{2}{n} \sum_{i, j} \bar{\sigma}\left(\sigma^{\prime}\left(E_{i}, E_{j}\right), \sigma^{\prime}\left(E_{i}, E_{j}\right)\right) .
\end{aligned}
$$

As we mentioned in Section $2, f: S^{m} \rightarrow S M(m+1)$ is of 1-type and $S^{m}$ is isometrically immersed in a hypersphere, say $W$, of $S M(m+1)$ centered at $I /(m+1)$ as a minimal submanifold.

The general assumptions we made in this paper are

(1) $x=f \circ \psi: M \rightarrow S^{m} \rightarrow S M(m+1)$ is of 2-type and

(2) $x=f \circ \psi$ is mass-symmetric, i.e., the center of mass of $M$ in $S M(m+1)$ is the center of the hypersphere $W$ in $S M(m+1)$, which means that $x_{0}=I /(m+1)$; and

(3) the immersion $\psi: M \rightarrow S^{m}$ is full, i.e., $\psi(M)$ is not contained in any great hypersphere of $S^{m}$.

Under these assumptions we have

$$
\Delta H=b H^{\prime}+\frac{b}{n} \sum_{i=1}^{n} \bar{\sigma}\left(E_{i}, E_{i}\right)+e\left(x-\frac{1}{m+1} I\right)
$$

where $b=\lambda_{p}+\lambda_{q}$ and $e=\lambda_{p} \lambda_{q} / n$. We put

$$
L=\sum \bar{\sigma}\left(E_{i}, D_{E_{i}} H^{\prime}\right) \text {. }
$$

Then, by using (2.6) and (3.8), we obtain 


$$
\begin{aligned}
g(\Delta H, L) & =-4 g(L, L)=-4 \sum_{i, j}\left\langle E_{i}, E_{j}\right\rangle\left\langle D_{E_{i}} H^{\prime}, D_{E_{j}} H^{\prime}\right\rangle \\
& =-4\left|D H^{\prime}\right|^{2} .
\end{aligned}
$$

On the other hand, (2.6), (2.7) and (3.9) imply

$$
g(\Delta H, L)=e g(x, L)=-e \sum\left\langle E_{i}, D_{E_{i}} H^{\prime}\right\rangle=0 .
$$

Therefore, from (3.11) and (3.12), we see that $\psi: M \rightarrow S^{m}$ has parallel mean curvature vector, i.e., $D H^{\prime}=0$. Thus, we have $\Delta^{D} H^{\prime}=\operatorname{tr}\left(\vec{\nabla} A_{H^{\prime}}\right)=0$.

For the immersion $x: M \rightarrow S^{m}$ we may regard the Weingarten map $A$ as a linear map from the normal bundle $T^{\perp} M$ into the space of selfadjoint endomorphisms $S_{n}(T M)$ of the tangent bundle $T M$ :

$$
A: T^{\perp} M \rightarrow S_{n}(T M)
$$

which carries $\xi \in T^{\perp} M$ onto $A_{\xi}$. On $S_{n}(T M)$ there is a canonical inner product defined by $\langle\langle B, C\rangle\rangle=(1 / n) \operatorname{tr}(B C)$ for $B, C \in S_{n}(T M)$. We say that the Weingarten map $A$ is homothetic if there exists a positive number $\rho$ such that $\left\langle\left\langle A_{\xi}, A_{\eta}\right\rangle\right\rangle=\rho\langle\xi, \eta\rangle$ for $\xi, \eta \in T^{\perp} M$. Submanifolds with conformal or homothetic Weingarten map were investigated in [2].

LeMma 1. Let $\psi: M \rightarrow S^{m}$ be a full isometric immersion. If $x=f \circ \psi$ is mass-symmetric and of 2-type, then

(1) the mean curvature vector of $\psi$ is parallel, i.e., $D H^{\prime}=0$,

(2) $\mathfrak{U}^{\prime}\left(H^{\prime}\right)=0$, i.e., $\sum \sigma^{\prime}\left(E_{i}, A_{H}, E_{i}\right)$ is parallel to $H^{\prime}$,

(3) $\left\|A_{H^{\prime}}\right\|$ is constant,

(4) the Weingarten map $A$ of $\psi$ is homothetic on $\left\langle H^{\prime}\right\rangle^{\perp}$, where $\left\langle H^{\prime}\right\rangle^{\perp}$ is the orthogonal complement of $\left\langle H^{\prime}\right\rangle=\operatorname{Span}\left\{H^{\prime}\right\}$, and

(5) the Ricci tensor $S$ of $M$ satisfies

$$
S(X, Y)=2 n\left\langle A_{H^{\prime}}, X, Y\right\rangle+k\langle X, Y\rangle
$$

for some constant $k$. ( $k$ depends only on $\lambda_{p}$ and $\lambda_{q}$ ).

Proof. Since $x=f \circ \psi: M \rightarrow S M(m+1)$ is assumed to be masssymmetric and of 2-type, $H^{\prime}$ is parallel in the normal bundle of $M$ in $S^{m}$. In particular, the length of $H^{\prime}$ is constant. Since $\Delta^{D} H^{\prime}=\operatorname{tr}\left(\bar{\nabla} A_{H^{\prime}}\right)=0$, (3.8) and (3.9) imply $\mathfrak{X}^{\prime}\left(H^{\prime}\right)=0$ and $\left\|A_{n+1}\right\|^{2}+3 n+4=b$. This proves (2) and (3).

From (2.6) and (3.8) we have

$$
\begin{aligned}
g(\Delta H, \bar{\sigma}(\xi, \eta))=[ & \left.4(n+1)+2 n\left\|H^{\prime}\right\|^{2}\right]\langle\xi, \eta\rangle \\
& -2 n\left\langle H^{\prime}, \xi\right\rangle\left\langle H^{\prime}, \eta\right\rangle-\frac{4}{n} \operatorname{tr}\left(A_{\xi} A_{\eta}\right)
\end{aligned}
$$


for any normal vector fields $\xi$, $\eta$ of $M$ in $S^{m}$.

On the other hand, (2.7) and (3.9) give

$$
g(\Delta H, \bar{\sigma}(\xi, \eta))=(2 b-e)\langle\xi, \eta\rangle .
$$

From (3.13) and (3.14) we find

$$
\begin{aligned}
\left\langle\left\langle A_{\xi}, A_{\eta}\right\rangle\right\rangle= & \frac{1}{4}\left[4(n+1)+2 n\left\|H^{\prime}\right\|^{2}+e-2 b\right]\langle\xi, \eta\rangle \\
& -\frac{n}{2}\left\langle H^{\prime}, \xi\right\rangle\left\langle H^{\prime}, \eta\right\rangle
\end{aligned}
$$

which proves the homotheticy of $A$ on $\left\langle H^{\prime}\right\rangle^{\perp}$.

From (2.6) and (3.8) we find

$$
\begin{aligned}
& g\left(\Delta H, \bar{\sigma}\left(E_{k}, E_{l}\right)\right)=\left[4(n+1)+\frac{4(n+1)}{n}+2 n\left\|H^{\prime}\right\|^{2}\right]\left\langle E_{k}, E_{l}\right\rangle \\
& +4\left\langle\sigma^{\prime}\left(E_{k}, E_{l}\right), H^{\prime}\right\rangle+\frac{4}{n} \sum_{i}\left\langle\sigma^{\prime}\left(E_{k}, E_{i}\right), \sigma^{\prime}\left(E_{l}, E_{i}\right)\right\rangle .
\end{aligned}
$$

From (2.6), (2.7) and (3.9) we get

$$
g\left(\Delta H, \bar{\sigma}\left(E_{k}, E_{l}\right)\right)=\left(2 b+\frac{2 b}{n}-e\right)\left\langle E_{k}, E_{l}\right\rangle .
$$

Since the Ricci tensor $S$ of $M$ satisfies

$$
\begin{aligned}
S\left(E_{k}, E_{l}\right)= & (n-1)\left\langle E_{k}, E_{l}\right\rangle-\sum_{\imath}\left\langle\sigma^{\prime}\left(E_{k}, E_{i}\right), \sigma^{\prime}\left(E_{l}, E_{\imath}\right)\right\rangle \\
& +n\left\langle\sigma^{\prime}\left(E_{k}, E_{l}\right), H^{\prime}\right\rangle,
\end{aligned}
$$

(3.16), (3.17) and (3.18) imply

$$
\begin{aligned}
& S\left(E_{i}, E_{j}\right)=2 n\left\langle A_{H}, E_{i}, E_{j}\right\rangle \\
& +\left[n(n+3)+\frac{n^{2}}{2}\left\|H^{\prime}\right\|^{2}+\frac{n e}{4}-\frac{b(n+1)}{2}\right]\left\langle E_{\imath}, E_{j}\right\rangle .
\end{aligned}
$$

This proves (5).

(Q.E.D.)

Remark 1. (i) It is not difficult to verify that if a submanifold $M$ of $S^{m}$ satisfies conditions (1)-(5) of Lemma 1 , then $x=f \circ \psi$ is mass-symmetric and it is of 1 or 2-type.

(ii) Lemma 1 was obtained in [9] in the special case when $M$ is a minimal submanifold of $S^{m}$. So Lemma 1 is a generalization of Ros' characterization theorem. 
By applying Lemma 1, we have the following,

Theorem 1. Let $\psi: M \rightarrow S^{m}$ be an isometric immersion of a compact Riemannian manifold such that the immersion is full. If $x=f \circ \psi$ is masssymmetric and of 2-type in $S M(m+1)$, then either

(a) $M$ is of 1-type in $E^{m+1}$ and so $M$ is minimal in a hypersphere of $E^{m+1}$ or

(b) $M$ is of 2-type in $E^{m+1}$ and mass-symmetric in $S^{m} \subset E^{m+1}$.

Proof. Under the hypothesis, Lemma 1 implies $D H^{\prime}=0 \mathfrak{U}^{\prime}\left(H^{\prime}\right)=0$ and $\left\|A_{H^{\prime}}\right\|$ being constant. Therefore, by applying Theorem 4.4 of $[6, \mathrm{p}$. 278], we conclude that either $M$ is of 1-type in $E^{m+1}$ or $M$ is mass-symmetric and of 2-type in $S^{m} \subset E^{m+1}$.

(Q.E.D.)

If $M$ is Einsteinian, then case (b) of Theorem 1 cannot occur. In fact, we have

Theorem 2. Let $\psi: M \rightarrow S^{m}$ be an isometric immersion of a compact Einstein manifold $M$ into $S^{m}$ such that the immersion is full. If $x=f \circ \psi$ is mass-symmetric and of 2-type, then either $M$ is minimal in $S^{m}$ or $M$ is minimal in a small hypersphere of $S^{m}$. In both cases, $M$ is of 1-type in $E^{m+1}$.

Proof. Under the hypothesis, statement (5) of Lemma 1 implies that $M$ is pseudo-umbilical in $S^{m}$. Moreover, from statement (1) of Lemma 1 , $M$ has parallel mean curvature vector $H^{\prime}$ in $S^{m}$. Thus, by applying Proposition 4.2 of [6, p. 133], we obtain the theorem.

(Q.E.D.)

We give the following lemma for later use.

LEMma 2. Let $M=S^{n}(r)$ be a small hypersphere of radius $r(r<1)$ of $S^{n+1}$. Then $M$ is of 2-type in $S M(n+2)$ via $f: S^{n+1} \rightarrow S M(n+2)$. Moreover, $M$ is mass-symmetric and of 2-type in $S M(n+2)$ if and only if $r^{2}=$ $(n+1) /(n+2)$.

Proof. Let $V_{i}$ be the eigenspace of $\Delta$ on $M$ with eigenvalue $\lambda_{i}$. Then we have $V_{1} V_{1} \subset V_{0}+V_{1}+V_{2}$. Without loss of generality we may assume that $M$ is given by the intersection of $S^{n+1} \subset E^{n+2}$ and the hyperplane $P$ of $E^{n+1}$ whose last coordinate is given by $\sqrt{1-r^{2}}$. Thus, $M=\left\{\left(y, \sqrt{1-r^{2}}\right)\right.$ $\left.\in E^{n+2} \mid y \cdot y^{t}=r^{2}\right\}$. Since the immersion $f: S^{n+1} \rightarrow S M(n+2)$ is defined by $f(u)=u^{t} \cdot u$ for $u \in S^{n+1}$, it is clear that $M$ is of 2-type in $S M(n+2)$ via $f$. Since $M$ is immersed in $S M(n+2)$ by $\left(y, \sqrt{1-r^{2}}\right)^{t} \cdot\left(y, \sqrt{1-r^{2}}\right)$, we see 
that the center of mass $x_{0}$ of $M$ in $S M(n+2)$ is proportional to the identity matrix $I$ of $S M(n+2)$ if and only if $r^{2}=(n+1) /(n+2)$. Moreover, in this case, we have $x_{0}=(1 /(n+2)) I$ which is exactly the center of the hypersphere $W$ which $S^{n+1}$ lies via $f$.

(Q.E.D.)

In the following three sections, we shall apply previous results to obtain some classifications results.

\section{$\S 4$. Hypersurface of $S^{m}$ which are of 2-type via $f$}

The main purpose of this section is to classify hypersurfaces of $S^{m}$ which are mass-symmetric and of 2-type via $f$.

Let $M=S^{p}\left(r_{1}\right) \times S^{n-p}\left(r_{2}\right)$ be the Riemannian product of two spheres with radii $r_{1}$ and $r_{2}$, respectively. Let $M$ be a hypersurface of $S^{n+1}=$ $S^{n+1}(1)$. Then we have $r_{1}^{2}+r_{2}^{2}=1$. We recall that

$$
\begin{aligned}
& \operatorname{Spec}\left(S^{p}\left(r_{1}\right)\right)=\left\{\bar{\lambda}_{k}=k(p+k-1) / r_{1}^{2} \mid k \geq 0\right\} \quad \text { and } \\
& \operatorname{Spec}\left(S^{n-p}\left(r_{2}\right)\right)=\left\{\lambda_{k}^{\prime}=k(n-p+k-1) / r_{2}^{2} \mid k \geq 0\right\} .
\end{aligned}
$$

Moreover, the coordinate functions of $x_{i}$ of $S^{p}\left(r_{1}\right)$ in $E^{p+1}$ are eigenfunctions with eigenvalue $\bar{\lambda}_{1}$ and the coordinate functions $y_{t}$ of $S^{n-p}\left(r_{2}\right)$ in $E^{n-p+1}$ are eigenfunctions with eigenvalue $\lambda_{1}^{\prime}$. Therefore, the coordinate functions of $M=S^{p}\left(r_{1}\right) \times S^{n-p}\left(r_{2}\right)$ in $S M(n+2)$ via $f$ are given by the following matrix

$$
\left[\begin{array}{c:c}
x_{i} x_{j} & x_{i} y_{t} \\
\hdashline x_{i} y_{t} & y_{t} y_{s}
\end{array}\right]_{\substack{1 \leq i, j \leq p+1, p \\
1 \leq s \leq n+1-p}} .
$$

So the coordinate functions of $M$ in $S M(n+2)$ are eigenfunctions on $M$ associated with at most three eigenvalues of $\Delta$ on $M$ given by $\bar{\lambda}_{2}, \lambda_{2}^{\prime}$ and $\lambda_{1}^{\prime}+\bar{\lambda}_{1}$.

Lemma 3. $\quad M=S^{p}\left(r_{1}\right) \times S^{n-p}\left(r_{2}\right)\left(r_{1}^{2}+r_{2}^{2}=1\right)$ is of 2-type in $S M(n+2)$ via $f$ if and only if either

(1) $r_{1}^{2}=(p+1) /(n+2)$ and $r_{2}^{2}=(n-p+1) /(n+2)$ or

(2) $r_{1}^{2}=(p+2) /(n+2)$ and $r_{2}^{2}=(n-p) /(n+2)$, or

(3) $r_{1}^{2}=p /(n+2)$ and $r_{2}^{2}=(n-p+2) /(n+2)$.

Proof. $M$ is of 2-type via $f$ if and only if two of $\bar{\lambda}_{2}, \lambda_{2}^{\prime}$ and $\lambda_{1}^{\prime}+\bar{\lambda}_{1}$ are equal. This implies the Lemma.

Lemma 4. $\quad M=S^{p}\left(r_{1}\right) \times S^{n-p}\left(r_{2}\right) \quad\left(r_{1}^{2}+r_{2}^{2}=1\right)$ is mass-symmetric in 
$S M(n+2)$ via $f$ if and only if $r_{1}^{2}=(p+1) /(n+2)$ and $r_{2}^{2}=(n-p+1) /$ $(n+2)$.

Proof. First we regard $M=S^{p}\left(r_{1}\right) \times S^{n-p}\left(r_{2}\right)$ as a submanifold in $E^{n+2}=E^{p+1} \oplus E^{n-p+1}$ in a natural way. It is easy to see that the center of mass of $M$ in $S M(n+2)$ via $f$ is given by

$$
\left[\begin{array}{cc}
\frac{r_{1}^{2}}{p+1} I_{p+1} & 0 \\
0 & \frac{r_{2}^{2}}{n-p+1} I_{n-p+1}
\end{array}\right\} \text {. }
$$

Thus, $M$ is mass-symmetric if and only if $(n-p+1) r_{1}^{2}=(p+1) r_{2}^{2}$. Because $r_{1}^{2}+r_{2}^{2}=1$, we obtain the Lemma.

Now, we give the following main result of this section.

Theorem 3. Let $\psi: M \rightarrow S^{n+1}$ be an isometric immersion of a compact $n$-dimensional Riemannian manifold $M$ into $S^{n+1}$. Then $x=f \circ \psi$ is masssymmetric and of 2-type if and only if either

(1) $M$ is a small hypersphere of $S^{n+1}$ with radius

$$
r=[(n+1) /(n+2)]^{1 / 2} \text {, or }
$$

(2) $\quad M=S^{p}\left(r_{1}\right) \times S^{n-p}\left(r_{2}\right)$ with $r_{1}^{2}=(p+1) /(n+2)$ and

$$
r_{2}^{2}=(n-p+1) /(n+2) \text {. }
$$

The immersions of $M$ into $S^{n+1}$ in (1) and (2) are given in natural way.

Proof. If $M$ is mass-symmetric and of 2-type in $S M(n+2)$ via $f$, then Lemma 1 implies that $D H^{\prime}=0,\left\|A_{H^{\prime}}\right\|$ is constant and the Ricci tensor $S$ of $M$ satisfies

$$
S(X, Y)=2 n\left\langle A_{H}, X, Y\right\rangle+k\langle X, Y\rangle,
$$

where $k$ is a constant. On the other hand, from Gauss' equation, we have

$$
S(X, Y)=(n-1)\langle X, Y\rangle+n \alpha^{\prime}\langle A X, Y\rangle-\left\langle A^{2} X, Y\right\rangle
$$

where $A$ is the Weingarten map of $M$ in $S^{n+1}$ Combining (4.2) and (4.3) we find $A^{2}+n \alpha^{\prime} A+(k+1-n) I=0$. This shows that $M$ has at most two distinct principal curvatures and the principal curvatures are constant. If $M$ has only one principal curvature, $M$ is a small hypersurface of $S^{n+1}$. In this case, Theorem 3 follows from Lemma 2 . If $M$ has two distinct principal curvatures, then $M$ is the product of two spheres. In this case, Theorem 3 follows from Lemma 3 and Lemma 4.

(Q.E.D.) 
Remark. Let $W$ be the hypersphere of $S M(n+2)$ in which $S^{n+1}$ is immersed as a minimal submanifold via $f$. Examples (2) and (3) of Lemma 3 give the first known examples of 2-type submanifolds in $W$ which are not mass-symmetric.

\section{§5. Submanifolds with maximal codimension}

Let $M$ be an $n$-dimensional submanifold of $S^{m}$. Consider the associated Weingarten map $A: T^{\perp} M \rightarrow S_{n}(T M)$ from the normal space of $M$ in $S^{m}$ into the vector bundle of self-adjoint endomorphisms of $T M$. In the vector bundle $S_{n}(T M)$ we consider the subbundle $M_{n}=\left\{B \in S_{n}(T M) \mid\right.$ trace $\left.B=0\right\}$. Then we have

$$
S_{n}(T M)=M_{n} \oplus R I_{n} .
$$

With respect to the usual inner product $\langle\langle\rangle$,$\rangle on S_{n}(T M)$, the subbundles $M_{n}$ and $R I_{n}$ are orthogonal. It is easy to see that the fibres of $S_{n}(T M)$ are of $\frac{1}{2} n(n+1)$-dimensional.

Lemma 5. Let $\psi: M \rightarrow S^{m}$ be an isometric immersion of a compact $n$-dimensional Riemannian manifold $M$ into $S^{m}$ such that the immersion is full. If $x=f \circ \psi$ is mass-symmetric and of 2-type, then we have $m \leq n(n+3) / 2$. In particular, if $m=n(n+3) / 2$, then $M$ is immersed as a minimal submanifold in a small hypersphere of $S^{m}$ via $\psi$.

Proof. Under the hypothesis, Lemma 1 implies that $M$ has parallel mean curvature vector in $S^{m}$. Thus, $M$ has constant mean curvature. If $M$ is minimal in $S^{m}$, then $A\left(T^{\perp} M\right) \subset M_{n}$. Since $\psi$ is full, statement (4) of Lemma 1 implies $m-n \leq n(n+1) / 2-1$ which gives $m \leq n(n+3) / 2-1$. Therefore, we may assume that $M$ has nonzero constant mean curvature in $S^{m}$. In this case, we obtain $m \leq n(n+3) / 2$. If $m=n(n+3) / 2$, then we see that $A: T^{\perp} M \rightarrow S_{n}(T M)=M_{n} \oplus R I$ is surjective. Since $A$ maps $\nu=\left\langle H^{\prime}\right\rangle^{\perp}$ onto $M_{n}$, we have $A\left(H^{\prime}\right) \in R I_{n}$. This shows that $M$ is pseudoumbilical in $S^{m}$. Because $M$ has parallel mean curvature vector $H^{\prime}$ in $S^{m}$, we conclude that $M$ lies in a hypersphere $S^{m-1}(r)$ of $S^{m}$ as a minimal submanifold. Since $M$ is not minimal in $S^{m}$, we have $r<1$. (Q.E.D.)

By applying Lemma 5 we may obtain the following.

Theorem 4. Let $\psi: M \rightarrow S^{n(n+3) / 2}$ be an isometric immersion of a compact, n-dimensional, Riemannian manifold $M$ into $S^{n(n+3) / 2}$ such that the 
immersion is full. If $x=f \circ \psi$ is mass-symmetric and of 2-type, then $M$ is a real-space-form which is immersed fully in a small hypersphere of $S^{n(n+3) / 2}$ as a minimal, isotropic submanifold.

Proof. Under the hypothesis, Lemma 5 implies that $M$ is immersed as a minimal submanifold in a small hypersphere $S^{n(n+3) / 2-1}(r)=S$ of $S^{n(n+3) / 2}$. Moreover, from Lemma 1 , we know that the Weingarten map $A$ of $M$ in $S$ is homothetic. Thus, for any fixed point $p \in M$, the Weingarten map at $p ; A(p): T_{p}^{\perp} M \rightarrow M_{n}(p)$ is an isomorphism. Since $A(p)$ is homothetic, we have

$$
\left\langle\left\langle A_{\xi}, A_{\eta}\right\rangle\right\rangle=c^{2}\langle\xi, \eta\rangle
$$

for some constant $c$. Let $v$ be a given unit vector in $T_{p} M$. We choose an orthonormal basis $B=\left\{e_{1}, \cdots, e_{n}\right\}$ such that $e_{1}=v$. Since $A(p): T_{p}^{\perp} M$ $\rightarrow M_{n}(p)$ is an isomorphism, there exists an orthonormal basis $\xi_{n+1}, \cdots$, $\xi_{n(n+3) / 2-1}$ in $T_{p}^{\perp} M$ such that, with respect to $B$, the associated Weingarten endmorphisms are given by

$$
\begin{aligned}
& A_{n+1}=c\left(\begin{array}{c|c}
-(n-1) a_{n-1} & 0 \\
\hline 0 & a_{n-1} I_{n-1}
\end{array}\right), \\
& A_{n+2}=c\left(\begin{array}{cc|c}
0 & 0 & 0 \\
0 & -(n-2) a_{n-2} & \\
\hline & 0 & a_{n-2} I_{n-2}
\end{array}\right), \\
& A_{2 n-2}=c\left(\begin{array}{c|c|c}
0 & 0 & 0 \\
\hline 0 & -2 a_{2} & \\
\hline 0 & a_{2} I_{2}
\end{array}\right), \\
& A_{2 n-1}=c\left(\begin{array}{cc|c}
0 & 0 & 0 \\
0 & -a_{1} & \\
\hline 0 & a_{1}
\end{array}\right),
\end{aligned}
$$

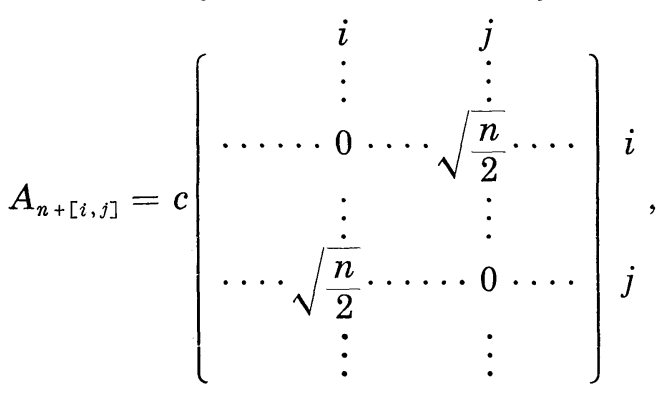


where $[i, j]=i+\frac{1}{2}(j-i)(2 n+1-j+i)-1, a_{n-h}^{2}=n /(n-k)(n-k+1)$; $1 \leq k \leq n-1$ and $1 \leq i<j \leq n$. From these we see that the second fundamental form $\bar{\sigma}$ of $M$ in $S$ satisfies $\|\bar{\sigma}(v, v)\|^{2}=(n-1) c^{2}$ which shows the isotropy of $M$ in $S$. The constancy of sectional curvature of $M$ follows from the equation of Gauss.

(Q.E.D.)

Remark. Isotropic isometric immersions from a real-space-form into another real-space-form have been studied by Itoh and Ogiue [8].

By a similar argument we have the following.

Theorem 5. Let $\psi: M \rightarrow S^{m}$ be an isometric minimal immersion of a compact, n-dimensional, Riemannian manifold such that the immersion is full. If $x=f \circ \psi$ is mass-symmetric and of 2-type, then $m \leq n(n+3) / 2-1$. In particular, if $m=n(n+3) / 2-1$, then $M$ is a real-space-form which is immersed as an isotropic submanifold.

Since this theorem can be proved in the same way as that of Theorem 4, so we omit it.

\section{§6. Classification of 2-type surfaces}

In this section we classify surfaces in $S^{m}$ which are mass-symmetric and of 2-type via $f$.

Theorem 6. Let $\psi: M \rightarrow S^{m}$ be an isometric immersion of a compact surface $M$ into $S^{m}$ such that the immersion is full. If $x=f \circ \psi$ is masssymmetric and of 2-type, then one of the following statements holds:

(1) $m=3$ and $M$ is immersed as a small hypersphere $S^{2}(r)$ with radius $r=\sqrt{3} / 2$;

(2) $m=3$ and $M$ is immersed as a Clifford (minimal) torus $S^{1}(1 / \sqrt{2}) \times S^{1}(1 / \sqrt{2})$ in $S^{3}$;

(3) $m=4$ and $M$ is immersed as a Veronese (minimal) surface in $S^{4}$;

(4) $m=5$ and $M$ is immersed as a Veronese (minimal) surface in a small hypersphere $S^{4}(\sqrt{5 / 6})$ of $S^{5}$.

The converse is also true.

Proof. Under the hypothesis, Lemma 1 implies that $M$ has parallel mean curvature vector in $S^{m}$. Thus, by applying a result of Chen and Yau (cf. [4, p. 106]), we have $m>3$ and either $M$ is a minimal surface of $S^{m}$ or $M$ is a minimal surface of a small hypersphere $S^{m-1}(r)$ of $S^{m}$, or $M$ lies in totally geodesic $S^{3}$ of $S^{m}$. If the later case holds, then $m=3$ since 
$\psi$ is full. In this case, Theorem 3 implies that either case (1) or case (2) occurs.

If $m>3$, then, by Lemma $5, m=4$ or $m=5$. If $m=4$, Theorem 5 and Theorem 2 of [8] imply that $M$ is a Veronese surface in $S^{4}$. If $m=5$, by using Theorem 4, we see that $M$ is immersed in a small hypersphere $S^{4}(r)$ of $S^{5}$ as a Veronese surface. Without loss of generality, we may assume that $S^{4}(r)$ is given by $u_{6}=\sqrt{1-r^{2}}$, where $\left(u_{1}, \cdots, u_{6}\right)$ are the Euclidean coordinates of $S^{5}$ in $E^{6}$. From direct computation, we see that the center of mass of $M$ in $S M(6)$ via $f$ is given by

$$
x_{0}=\left(\begin{array}{c:c}
\frac{r^{2}}{5} I_{5} & 0 \\
\hline 0 & 1-r^{2}
\end{array}\right) \text {. }
$$

Since $M$ is mass-symmetric in $W \subset S M(6)$, we have $x_{0}=I / 6$. Thus, we see that $M$ is mass-symmetric in $S M(6)$ if and only if $r^{2}=5 / 6$.

The converse follows from direct computation.

(Q.E.D.)

\section{REFERENCES}

[1] Barros, M. and B. Y. Chen, Classification of stationary 2-type surfaces of hyperspheres, C.R. Math. Rep. Acad. Sci. Canada, 7 (1985), 309-314.

[2 ] Barros, M. and B. Y. Chen, Finite type spherical submanifolds, Proc. II Intern. Symp. Diff. Geom., Lecture Notes in Math., Springer-Verlag, 1209 (1986), 73-93.

[ 3 ] Barros, M. and A. Ros, Spectral geometry of submanifolds, Note Mat., 4 (1984), $1-56$.

[4] Chen, B. Y., Geometry of submanifolds, M. Dekker, 1973,

[5] Chen, B. Y., On total curvature of immersed manifolds, IV, Bull. Math. Acad. Sinica, 7 (1979), 301-311; — VI, ibid, 11 (1983), 309-328.

[6] Chen, B. Y., Total mean curvature and submanifolds of finite type, World Scientific, 1984.

[ 7 ] Chen, B. Y., 2-type submanifolds and their applications, Chinese J. Math., 14 (1986), 1-14.

[8] Itoh, T. and K. Ogiue, Isotropic immersions, J. Differential Geom., 8 (1973), 305-316.

[9] Ros, A., Eigenvalue inequalities for minimal submanifolds and $P$-manifolds, Math. Z., 187 (1984), 393-404.

[10] Sakamoto, K., Planar geodesic immersions, Tôhoku Math. J., 29 (1977), 25-56.

[11] Takahashi, T., Minimal immersions of Riemannian manifolds, J. Math. Soc. Japan, 18 (1966), 380-385.

M. Barros

Departamento de Geometria y Topologia

Universidad de Granada

18071-Granada, Spain 
B.-Y. Chen

Department of Mathematics

Michigan State University

East Lansing, Michigan 48824

USA 